\title{
TOPO-NET SPATIAL ENTITY RELATIONSHIP MODEL FOR GEOGRAPHIC INFORMATION SYSTEM APPLICATIONS
}

\author{
Barkha Bahl $^{1}$, Vandana Sharma ${ }^{2}$ and Navin Rajpal ${ }^{3}$ \\ ${ }^{1}$ Department of Information Technology, Guru Gobind Singh Indraprastha University, \\ New Delhi, India \\ Barkha69@rediffmail. com \\ ${ }^{2}$ Department of Geographic Information Systems, National Informatics Center, New \\ Delhi, India \\ Sharma.vandana@nic.com \\ ${ }^{3}$ Department of Information Technology, Guru Gobind Singh Indraprastha University, \\ New Delhi, India \\ navin@yahoo.com
}

\begin{abstract}
Rapid development of Geographic Information System (GIS) has given rise to great amount of spatial database, around which a large number of GIS applications are being created. A GIS based network application for planning and management being developed in the country has typical requirement for representation of datasets at primary level .This paper examines the representation of such datasets through ER and EER models and summarizes their limitation. In order to represent the evolving database design for GIS based network application Topo-Net Spatial ER was evolved and proposed with focus on representation of spatial, topological, network and generalization aspects and has led to successful conceptual database design. The representation have been achieved through moderating EER Model with topological and network concepts and hence termed as Topo-Net Spatial ER model. The approach and representations of the same are described in the present paper. The approach and representations of the same are described in the present paper and is being demonstrated through a case study for Optical Fiber Cable Network.
\end{abstract}

\section{KEYWORDS}

Data Model, Entity, Network, Optical Fiber, Relationship, Spatial Data, Topology.

\section{INTRODUCTION}

The term modeling refers to the manner in which the data is structured and stored in an information system. Many data models have been proposed which can be categorized according to the types of concepts they use to describe the database structure. High-level or conceptual data models provide concepts that are close to the way many users perceive data, whereas low level or

DOI: $10.5121 / \mathrm{ijdms.2011.3403}$ 
International Journal of Database Management Systems ( IJDMS ) Vol.3, No.4, November 2011

physical data models provide concepts that describe the details of how data is stored in the computer[1]. The third one is external or view level which is typically implemented using representational data model, these include the widely used relational, network and hierarchical models. In this paper the emphasize will be on conceptual schema, which describes the structure of whole database for a community of GIS users. In the Geographic Information Systems (GIS) community the concept of data modeling is well developed and integrated in the design of databases and application.

The data models in geographic databases or the GIS Data Model allows the geographic features in real world locations to be digitally represented and stored in a database so that they can be presented in a map form, and can also be worked with and manipulated to address some problem.A GIS System takes spatial and non-spatial data input, which is kept in a geographic database. This is constantly analyzed and transformed. Any query input provided by the user is answered by displaying and reporting the results.

The recent developments in GIS has lead Software Engineers towards effective and efficient use of databases by defining the relationship between different entities, which can be possible by defining the model of the databases properly. Efficient database designing will lead to better results. Conventional data models like ER [13] and EER[1] are suitable for representing entities and their relationships for commertial applications, where as various GIS specific data models like MADS[2,17] , ChronoGeoGraph[4] , Temporal EER models[15] etc are used to represent spatio-temporal features among the entities. GeoOOA[5] model can be used for representing network and topological features for object oriented representations. Relational representations of the network and topological features require more specific representations . Hence, to overcome the deficiency of representing geo-primitives and GIS-specific requirements in conventional ER and GIS specific models, we proposed Topo-Net Spatial ER model for its more adequate treatment of GIS -specific requirements.

The paper will focus on the existing ER models and the proposed Topo-Net Spatial Entity Relationship model. The second section will focus on the existing ER models; third section represents various proposed notations for spatial entity, relationship type, cardinality ratio and topological ,network representations ; fourth section will discuss the case study for optical fibre cable network representing its conceptual design through the proposed Topo-Net Spatial Entity Relationship model. Fifth section concludes the paper.

\section{EXISTING ENTITY RELATIONSHIP AND ENHANCED ER MODELS}

\subsection{CONVENTIONAL CONCEPTUAL MODELS}

Entity relationship(ER) model[13] is used to represent the relationship between entities and is a basic tool in database design. This model does a good job of capturing and representing the basic semantics of many different situations. However, the model, is not capable to capture more domain semantics for modern applications[1,7], hence more domain semantics are included in EER models[1] where an enhancement has been made through representation of generalization/ specialization, aggregation and classification in ER model. E-R models have a layered approach to organizing information in that the basic components of an E-R model, attributes, entities and relationships can only be combined in certain ways, on the other hand ,the extended entityrelationship mode[14] is decomposed according to a set of basic entity-relationship constructs, and these are transformed into candidate relations via entity relations, extended entity relations 
International Journal of Database Management Systems ( IJDMS ) Vol.3, No.4, November 2011

and relationship relations which reduce the number of data dependencies and maintains data integrity through normalization.

It has been observed that the above mentioned ER, EER models can represent traditional database design for conventional commercial applications and are not effectively suitable for GIS applications as they are incapable to represent spatial aspects. Various GIS specific conceptual models have been studied and discussed in section 2.2.

\subsection{GIS SPECIFIC CONCEPTUAL MODELS}

MADS is a spatio-temporal data model. It is a framework suitable for vector data for use at different resolution levels. The papers[2,17] talk about MADS data modeling, data matching from different data sets, and data utilization supporting multiple representations. It belongs to the family of entity relationship data models extended to supports the main concepts of object oriented and spatio-temporal features to be represented in conceptual database design. Remodeling of ER models into MADS has reduced the number of object and relationship types by a factor of $23 \%$ compared to ER models.

ChronoGeoGraph(CGG)[4] is another spatio-temporal model that pairs the classical features of the EER model with a large set of spatial and temporal constructs . It introduces spatial attributes that take their value over a geometry type and can be associated with spatial and non-spatial entities. CGG model is being extended so that multiple representations of topological relations can be dealt with[3]. Topological relations allow us to constrain the relationship between the geometries of pairs of spatial entities. Moreover it makes it possible to pair the spatial entity with multiple viewpoints, shapes and resolution by means of suitable primitives for cartographic specialization. Both MADS and CGG Model lacks to incorporate either the topological or the network or both the aspects of GIS applications.

Several models E.g. GeoOM[21], MODUL-R[22], and SPATIAL E-R model[9], Spatially Enhanced EER Model(SEER)[12] only supports the representation of spatial information, similarly temporal model e.g. Temporal Entity-Realtionship Model(TERM)[16], Temporal EER Model(TEER)[14],Relationships, Attributes, Keys and Entities Model(RAKE)[19] supports the association of time with objects, relationships and attributes. Whereas, in a real life for a GIS application, apart from Spatial, temporal and spatio-temporal scenario, topological and network scenarios are also frequently encountered. GeoOOA[5] Object-oriented Analysis for GIS overcomes the deficiencies of conventional spatio-temporal and object oriented analysis model by adding suitable domain tailored primitives such as topological, network etc. but is applicable for object - oriented analysis only.

In conclusion, none of the above mentioned spatial and temporal models satisfied the representation of topological and network Geo-primitives for relational GIS applications appropriately. This prompted the development of Topo-Net Spatial Entity Relationship model for representing topological, network and generalization features fulfilling the simplicity and comprehensiveness criterias, where simplicity of the model talks about easiness of use and understanding and comprehensiveness means the direct representation of topological and network aspects in the model. The Topo-Net spatial ER model has been designed through the proposed notations for a GIS application . 
International Journal of Database Management Systems ( IJDMS ) Vol.3, No.4, November 2011

\section{PROPOSED NOTATIONS}

The proposed notations for representing a Topo-Net Spatial ER model retains the flavor of EER Model, but the new representations have been achieved through moderating EER Model with spatial aspects. The spatial entity, relationships: topological and network, relationship types, generalization, aggregation and other related icons have been expressed in figure 2 and figure 3 respectively.

\subsection{NOTATIONS FOR SPATIAL ENTITY AND CARDINALITIES}

Spatial entities can be represented using the rectangular box with point, line, polygon symbols embedded inside the rectangular box as shown in Figure 1.

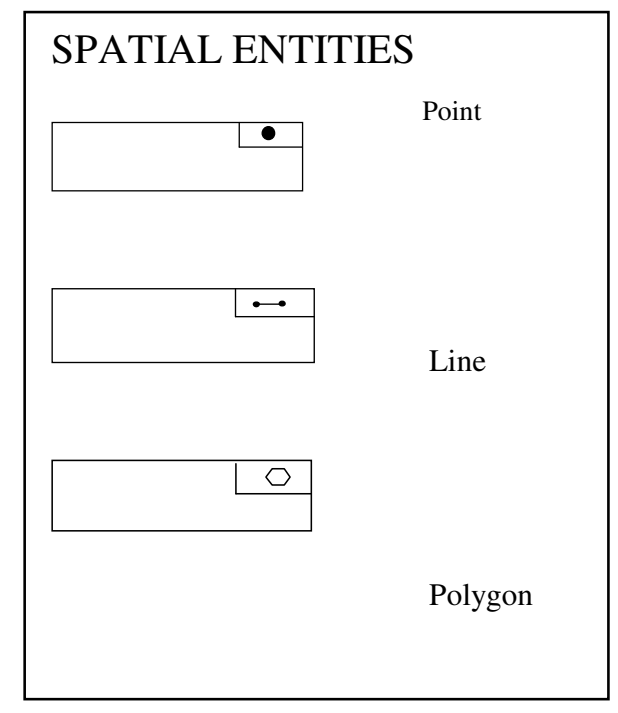

Figure 1. Notations for Spatial Entity

Lines express cardinalities based on an intuitive notation: dotted means optional, solid line with * means multivalued . Figure 2., Represents $1 \ldots \ldots 1,0 \ldots \ldots 1, \mathrm{~m} \ldots \ldots \ldots$.... etc. cardinalities.

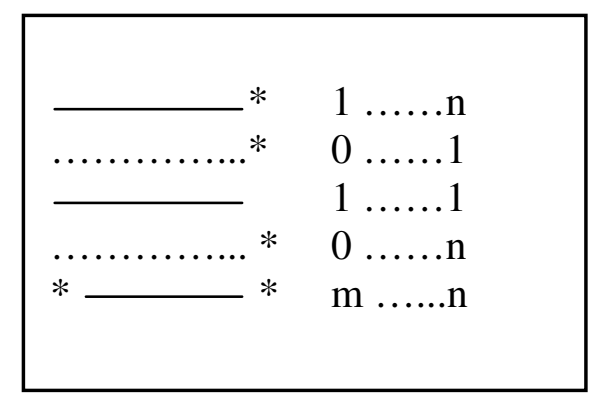

Figure 2. Notations For Cardinalities

\subsection{NOTATIONS FOR RELATIONSHIPS}

The relationships can be represented in the model using below mentioned notations: 
International Journal of Database Management Systems ( IJDMS ) Vol.3, No.4, November 2011

- $\quad$ Relationship type is expressed with in a circle.

- $\quad$ Generalization/specialization type can be represented with triangle inside a circle.

- $\quad$ Symbolic notations for fully connected and partially connected relationships have been represented in Figure. 3.

○ "fully connected to " relationship is being represented using solid circle notation,

○ "partially connected to" relationship is being represented using doted circle notations.

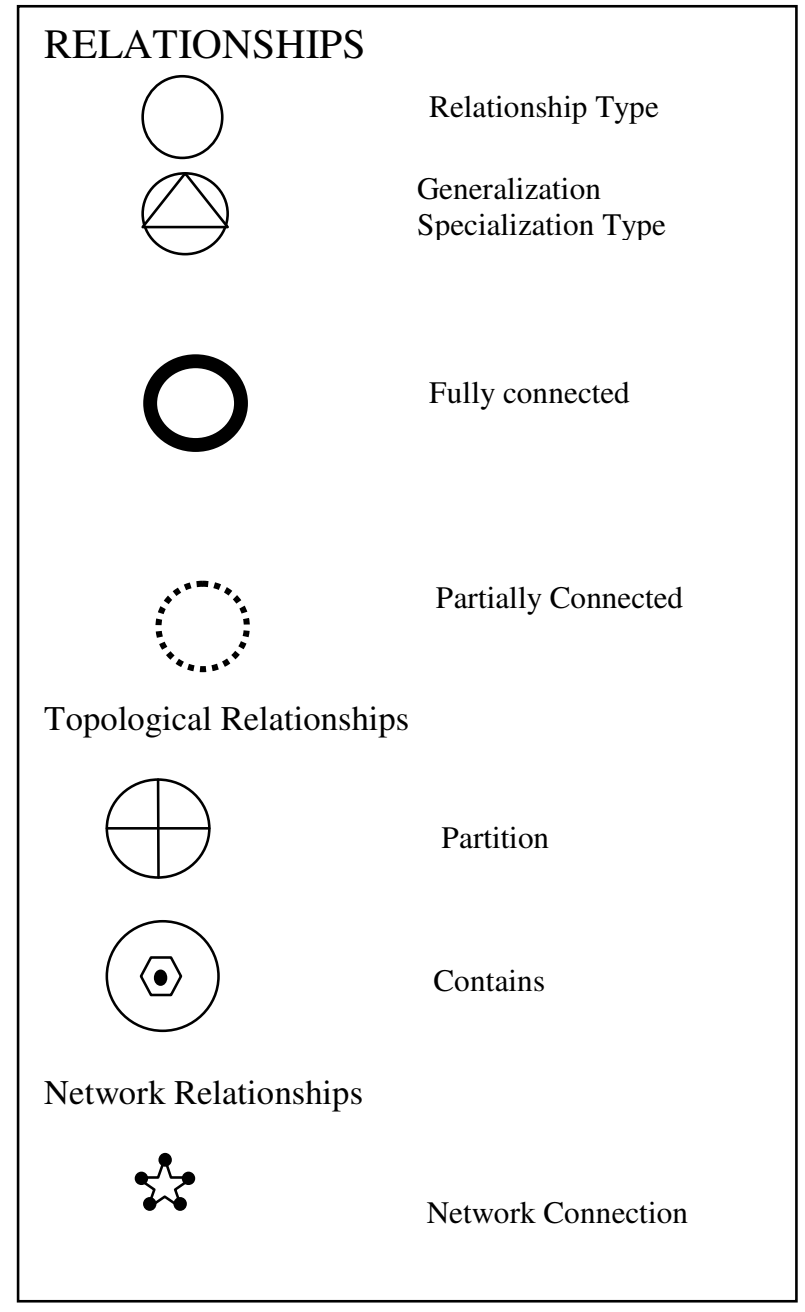

Figure 3. Notations for Relationships

\subsubsection{TOPOLOGICAL AND NETWORK RELATIONSHIPS}

Our first scenario concerns an application domain where entities are arranged according to topological whole-part relationships with certain topological constraints GeoOOA[5]. A typical example is the partition relationship and contains relationships.

For a containment structure, the geometry of the whole contains the geometry of all its parts. The same has been presented with

(๑) symbolic representation. 
International Journal of Database Management Systems ( IJDMS ) Vol.3, No.4, November 2011

The partition structure is a containment structure with two additional conditions: the whole and its parts belongs to the same spatial entity type and the geometries of the part forms a partition of the geometries of the whole with symbolic notation

The second scenario deals with networks where entities are connected through links. A network with spatial entities is called spatially embedded network. In the Topo-Net Spatial ER model of the optical fiber cable network diagram (Figure 5) following network relationship types have been represented.

"recursively connected to" relationship is proposed to represent the network relationship among entities of same type. This notation better explains and represents the recursive relationship between same entity types than the traditional ER Diagram. In OF cable network, Nodes are connected to nodes itself and it clearly maintains network among various nodes(points) and is represented with network symbol

\section{CASE STUDY FOR GIS APPLICATION - OPTICAL FIBER CABLE NETWORK'S CONCEPTUAL MODEL}

A GIS based application for network planning and management is being developed by the National Informatics center, Dept. of Information Technology, Govt. of India. Mapping of fiber optical cable network at its preliminary stage has been taken up at 1:50,000 scale. Long distance optical fiber mapping completed which is estimated over $1.5 \mathrm{~L} \mathrm{Km}$ for one of the largest telecom service provider in the country. The application has number of aspects on representations of data. Entities that need to be considered includes long distance optical fiber cable routes termed as segment, switching service area, optical fiber stations or exchanges etc. In order to represent these entities and their relationships, ER[13], EER[15], Spatial ER[9], and various other Models were considered such as GeoOM[21], MODUL-R[22], MADS[17] etc, and was found that they all have limitations to define representation of above mentioned GIS application. In order to overcome these difficulties Topo-Net Spatial ER Model is designed. The representation has evolved successfully fulfilling the objective of the problem.

Management of telecom infrastructure is undertaken through a typical hierarchical structure defining various areas of the country as regions such as state, district, sub district, gram panchayat etc. Optical fiber is used as a medium for telecommunication and networking because it is flexible and can be bundled as cables [11]. It is especially advantageous for long-distance communications, because light propagates through the fiber with little attenuation compared to electrical cables. The long distance cable is the backbone route which in turn shall get connected to optical fiber and further levels such as circles, secondary switching area etc.

The problem currently is to identify and represent various spatial entities and further to define and represent effectively topological, network and generalization relationships among them.

GIS based Optical fiber cable network database is designed to improve reliability, efficiency and quality of network management system.

The long Optical fiber cable connects large number of circles which covers various states and it is a national ring passing through many districts , Secondary Switching Area (SSA's), Sub District Charging Area (SDCA's) etc. The O/F network and maps proposes to specify the connection information, fault information and displays it on user request. Traditional network management systems provide real-time monitoring and management capabilities which are 
International Journal of Database Management Systems ( IJDMS ) Vol.3, No.4, November 2011

limited to providing details on faults and performance issues etc. and lack to document physical location. However, Geographical information systems based network management system , on the other hand, support detailed documentation of the physical network to help service provider to visualize the route through maps and high resolution satellite images and understand constraints of the locations that needs to be negotiated with for maintenance of network. The network spans a wide geographical area and run for hundreds and even thousands of miles.

\subsection{REQUIREMENTS FOR AN OPTICAL FIBER CABLE NETWORK DATABASE}

A common long distance optical fiber cable network database in the subscriber loop operations must be able to represent and manipulate large amount of datasets for an application. The Optical fiber cable network has entities that include

- Segments(long distance cable route)

$\circ$ Number of fibres running across that route (capacity)

○ Type Of Fiber (Fibre _type can be of 6,12,24,36,48 fiber)

- Nodes ( Long Distance cable connects various states, districts, sub districts, Gram Panchayat etc either directly or indirectly) and if can be named as

- Circles

○ Secondary switching area(SSA)

- Sub District Charging Area (SDCA)

$\circ$ Exchanges

The more challenge is to deliberate on the topological, network and generalization relationships among the above mentioned entities. The same has been explained in section 4 .

\subsection{ARCHITECTURE OF FIBER OPTICAL CABLE COMMUNICATION NETWORK}

The connection of fiber optical cable network for telecommunication is flexible, efficient and effective. The long distance cables connect primary rings consisting of circles, which in turn is connected to secondary ring comprising of SSA and connected to SDCA. Secondary ring is further connected to tertiary ring consisting of various exchanges. Finally through Exchanges subscriber can subscribe the connection to customers. Figure 4 depicts the hierarchical view of connections between the identified entities. 
International Journal of Database Management Systems ( IJDMS ) Vol.3, No.4, November 2011

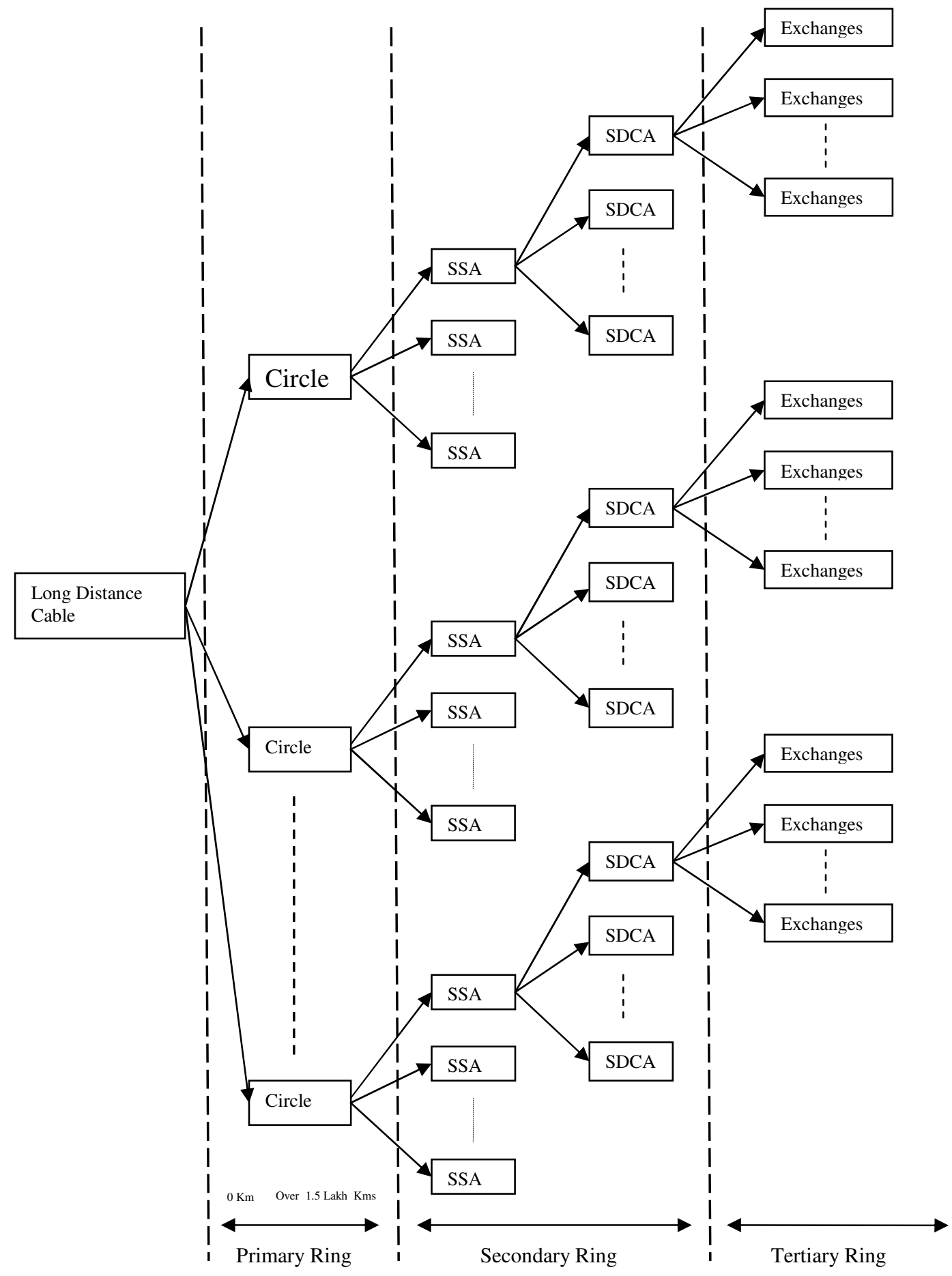

Figure 4. Long distance OF Connection to Gram Panchayat (Architecture) 
International Journal of Database Management Systems ( IJDMS ) Vol.3, No.4, November 2011

\subsection{CONCEPTUAL MODEL - REPRESENTATION THROUGH TOPO-NET SPATIAL ER MODEL}

On the basis of the requirements and the proposed notations Figure 5. represents the Topo_net Spatial ER model for Optical Fiber Cable Network .

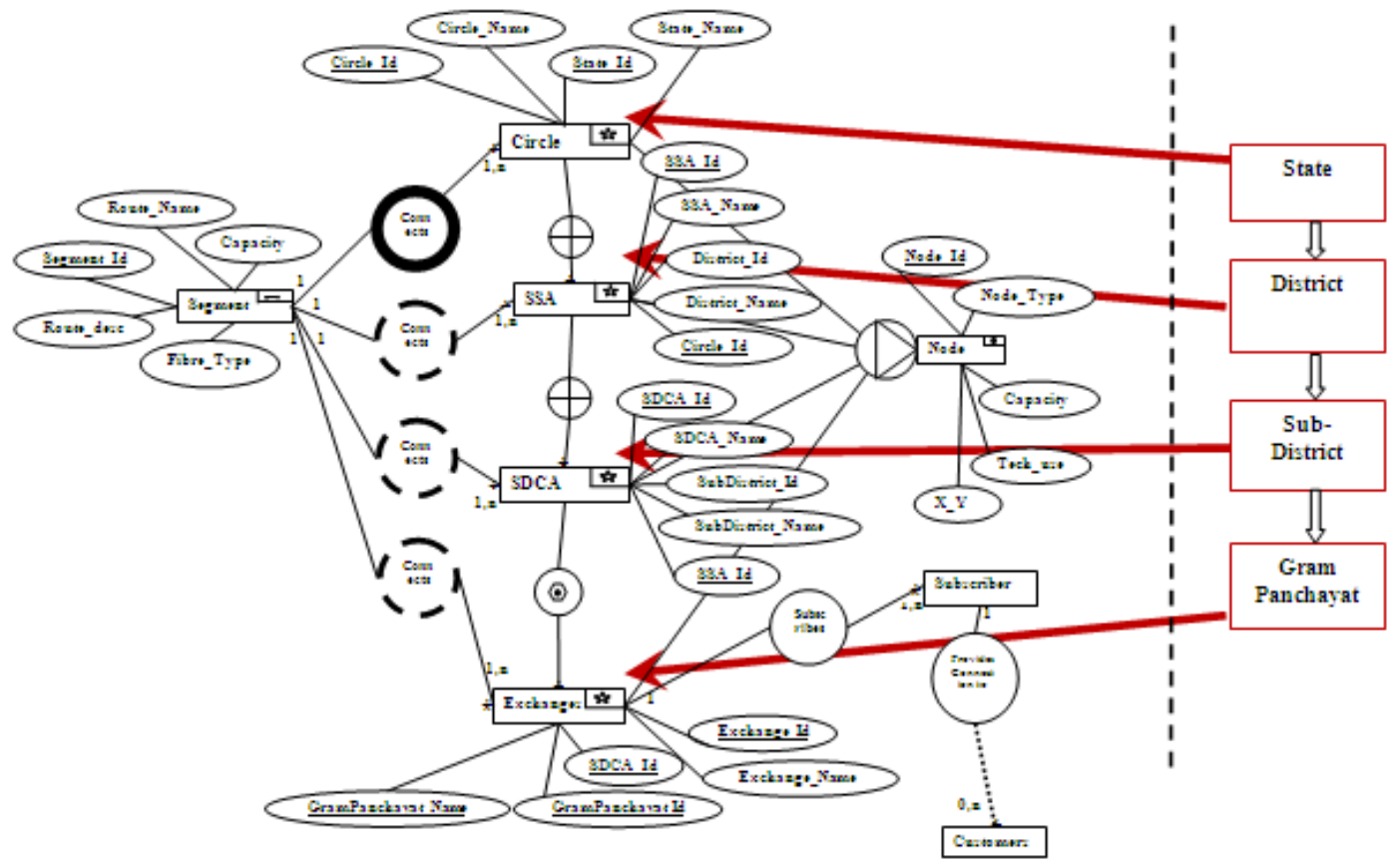

Figure 5. Topo-Net Spatial ER Model for Optical Fiber Cable Network

\subsubsection{NOTATIONS FOR RELATIONSHIPS}

The relationships can be represented in the model using below mentioned notations:

- Relationship type is expressed with in a circle. e.g. "subscribes" and "provides connection" relations between exchanges, subscriber and customer entities as shown in Figure 3 and Figure 5.

- Generalization/specialization type can be represented with triangle inside a circle. Since Nodes in the optical fiber cable network can be circle, SSA, SDCA or Exchanges, hence can be represented as generalization relationship as shown in Figure. 3 and Figure. 5.

- Symbolic notations for fully connected and partially connected relationships have been represented in Figure. 3.

○ "fully connected to " relationship is being represented between segment and circle using solid circle notation, since circles are connected via segments .i.e. long distance cable directly as shown in Figure. 5.

○ "partially connected to" relationship is being represented between segment and SSA's, SDCA's and Exchange's, through dashed circles, since SSA,SDCA, exchanges may or may not be connected to long distance cable i.e. segment directly as shown in Figure. 5. 
International Journal of Database Management Systems ( IJDMS ) Vol.3, No.4, November 2011

\subsubsection{TOPOLOGICAL AND NETWORK RELATIONSHIPS}

Our first scenario concerns an application domain where entities are arranged according to topological whole-part relationships with certain topological constraints GeoOOA [5]. A typical example is the partition relationship and contains relationships.

- $\quad$ For a containment structure, the geometry of the whole contains the geometry of all its parts. The same has been presented in Figure 5 between SDCA and exchanges with (๑) symbolic representation.

- The partition structure is a containment structure with two additional conditions: the whole and its parts belongs to the same spatial entity type and the geometries of the part forms a partition of the geometries of the whole.(depicted in Figure 5 among circles , ssa's and sdca's with symbolic notation).

The second scenario deals with networks where entities are connected through links. A network with spatial entities is called spatially embedded network. In the Topo-Net Spatial ER model of the optical fiber cable network diagram (Figure 5) following network relationship types have been represented.

- $\quad$ "recursively connected to" relationship is proposed to represent the network relationship among entities of same type. This notation better explains and represents the recursive relationship between same entity types than the traditional ER Diagram. In OF cable network, Nodes (circles, SSA,SDCA, etc) are connected to nodes itself and it clearly maintains network among various nodes(points) and is represented with network symbol ?or For an instance SSA are connected to SSA itself and forms a network of SSA's at District level. Similarly circle forms a network at State level, SDCAs form a network at Sub-District level and Exchanges forms a network at Gram-Panchayat level.

Comparing the Topo-Net Spatial ER model's representation of the Optical Fiber cable network with the ER/EER or spatial temporal representation reveals that through the proposed notations for spatial entities and relationship types the model gains clarity as well as expressiveness from the new primitives hence is able to fulfill the simplicity criteria. In contrast to the various EER model, relevant topological ,network and spatial information is directly expressed within the model showing the comprehensiveness of the model. Besides an improved graphical representation the textual specification benefits from keywords referring to predefined topological relationships and network domain specific contents along with constraints have also been expressed effectively and addition of these features to database can be performed easily.

\section{CONCLUSION}

This paper proposes a sound basis for the development of conceptual model for representing topological, network and spatial features for GIS applications. Indeed, an analysis of existing models shows that such a basis is weakly defined. The proposed Topo-Net Spatial ER model supports topological, network and Generalization features effectively meeting the specified criteria. The model is far more flexible, simple and comprehensive than what many other model offer. Topo-Net Spatial ER is developed in an application framework and has been used for modeling optical fiber cable network. Finally, using Topo-Net Spatial ER model led the application designers to discover the importance of topological, network and generalization features within their application. 
International Journal of Database Management Systems ( IJDMS ) Vol.3, No.4, November 2011

\section{REFERENCES}

[1] Antonio Badia, (2004),’Entity-relationship modeling revisited”, SIGMOD Record, Vol. 33, No. 1 pp 77- 82 .

[2] Christine Parent, Stefano Spaccapietra, Esteban Zimanyi, (1999), "Spatio-temporal conceptual modelsz data structures + space + time", GIS '99 Proceedings of the 7th ACM international symposium on Advances in geographic information systems, pp 26-33.

[3] Donatella Gubiani, Angelo Montanari, (2008), “A conceptual spatial model supporting topologicallyconsistent multiple representations”, In ACM. GIS'08, November,pp 5-7.

[4] Donatella Gubiani, Angelo Montanari, (2007), "ChronoGeoGraph: an expressive spatio-temporal conceptual model”, Proc. of the 15th Italian Symposium on Advanced Database Systems(SEBD), pp. $160-171$.

[5] Georg Kosters, Bernd-Uwe Pagel, Hans-Werner, (2006), “ GeoOOA: object-oriented analysis for geographic information systems”, IEEE proceedings of ICRE, pp 245-253.

[6] Gregor Engels, Martin Gogolla, Uwe Hohensteen, Klaus Hulsmann, Perdita Lohr-Richter, Gunten Saake, Hans-Dieter Ehrich,(1992), “Conceptual modelling of database applications using an extended ER model”, Data and Knowledge Engineering, Vol. 9, issue 2, pp. 157-204.

[7] Joan Peckham, Fred J. Maryanski, (1988), "Semantic data models", ACM computing surveys, Vol. 20, issue 3 pp 153-188.

[8] J.W. Van Roessel, (1987),'Design of a spatial data structure using the relational normal form”, In'1 Journal of Geographical Information System, pp.1-33.

[9] Li Su, Yijin Chen, Danhui Zhou, (2006), "Conceptual modeling of spatial database based on geographic ontology" Geoscience and Remote Sensing Symposium, IGARSS. IEEE International Conference. pp 1584-1586

[10] Manfred R. Klopprogge, "TERM : An approach to include the time dimension in the entity relationship model”, Proc. of the Second Int'l Conf. Entity relationship approach, pp.477-512

[11]NTT Science and Core Technology Laboratory Group, 2006.14 Tbit/s over a single optical fiber: successful demonstration of world's largest capacity. In Press release. http://www.ntt.co.jp/news/news06e/0609/060929a.html

[12] P.G.Firn, (1994), "An extended entity relationship model applicable to the design of spatially reference databases", Ph.D Dissertation, Dunedin, University of Otago.

[13] Peter Pin-Shan Chen, (1976), "The entity-relationship model-toward a unified view of data", Association for Computing Machinery Transactions on Database Systems, 1-3.

[14] Ramez Elmasri, Gene T.J.Wuu, (1990), "A temporal model and query language for ER databases", Proc. of the Sixth Int'l Conf. Data Eng. , pp. 76-83.

[15] Ramez Elmasri, Shamkant B. Navathe, (2009), “The Enhanced Entity -Relationship Model”, Fundamentals of Database Systems, 5th Edition, pp 103-131.

[16] Ralf Hartmut Guting, FernUniversitat Hagen, Michael H.Bohlen, Martin Erwig, Christian S. Jensen, Nikos A. Lorentzos, Markus Schneider, Michalis Vazirgiannis, (1998), ”A foundation for representing and querying moving objects", Informatik-Report 238, Fern Universitaet Hagen.

[17] Sandrine Balley, Christine Parent, Stefano Spaccapietra, (2004), "Modelling geographic data with multiple representations” Int. J. Geographical Information Science. Vol. 18 , No. 4, 327-352.

[18] Shao Quanqing, Zhou Chenhu, (1998), "The study on E-R method of data modeling in marine fishery", Geographical Research, Beijing, China, 17(sup), pp.108-115. 
International Journal of Database Management Systems ( IJDMS ) Vol.3, No.4, November 2011

[19] Stephen Ferg, (1985), "Modelling the time dimensions in an entity-relationship diagram", Proc. of the Fourth Int'l Conf. Entity -Relationship Approach, pp. 280-286.

[20] Toby J. Teorey, Dongqing Yang, James P. Fry, (1986), "A logical design methodology for relational databases using the extended entity-relationship model", ACM -Computing Survey(CSUR), Vol. 18, Issue 2, pp 418-429.

[21] Tryfona N., Pfoser D., Hadzilacos T., 1997, "Modeling behavior of geographic objects: an experiment with the object modeling technique", Pro. of the Ninth Int'l Conf. Advanced Information Systems Engineering, CAiSE'97, Spinger-Verlag, pp 124-139.

[22] Yvan Bedard, Cloude Caron, Zakaria Maamar, Bernard Moulin \& Denis Valliere, (1996), "Adapting data models for the design of spatio-temporal databases", Computer Environment and urban Systems, 20(1) pp 19-41.

\section{Authors}

Mrs. Barkha Bahl, received her MCA degree from Banasthali Vidyapeeth. She is Research Scholar with Guru Gobind Singh Indraprastha University, New Delhi, India. She has teaching experience of seventeen years and research experience of six years. Presently, working as Reader and Course Coordinator (MCA Programme), Delhi Institute of Advanced Studies, Affiliated to Guru Gobind Singh Indraprastha University, New Delhi. Guided several MCA students for developing research oriented projects. Five research papers/Articles have been published in national / international journals/proceedings. Her research area is - Databases and Geographic Information Systems.

Dr. Vandana Sharma, received her Master degree in Physics from Bhopal University, and Doctorate in Physics and Astrophysics from Delhi University,India. She joined at National Remote Sensing Centre (NRSC), a unit of Indian Space Research Organization in 1981 and worked till 1986. Then she joined in National Informatics Centre (NIC) and currently she is working in the capacity of Deputy Director General at NIC.

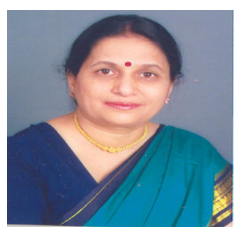

Dr. Navin Rajpal is a Professor with the University School of Information Technology, Guru Gobind Singh Indraprastha University since Sept. 2004. He received his B.Sc. Engineering Degree in Electronics \& Communication from Regional Engineering College, Kurukshetra in 1987, M.Tech. \& Ph.D. degree from Department of Computer Science \& Engineering, I.I.T., Delhi in 1991 and 1999 respectively. He worked as Reader at USIT GGSIP University from 2000-2004. Prior to this he worked as Assistant Professor at CRSCE Murthal from 1996-2000. He also worked as Scientific Officer at

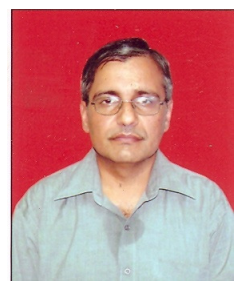
CARE, IIT Delhi from 1987-1995.He published more than 50 research papers in Journals and Conference Proceedings. Worked on 7 Sponsored and 2 Consultancy projects and released 3 Softwares for copyright at IIT Delhi. Supervised several M.Tech dissertations and one Ph.D. student. Currently supervising seven Ph.D. students. His research areas include Computer Graphics, Digital Image Processing, Pattern Recognition, Geographic Information System, Artificial Neural Networks, Data Structure, Algorithms Analysis and Design and Digital Hardware Design 